Pimenta, R.A.; Poeta, L.S.; Basso, L.; Mariano, M. y Rosa Neto, F. (2021) Association Between Specific Areas of Development and Dyspraxia in Preschool Children. Revista Internacional de Medicina y Ciencias de la Actividad Física y el Deporte, vol. 21 (82) pp. 403-418 Http://cdeporte.rediris.es/revista/revista82/artasociacion1252.htm

DOI: https://doi.org/10.15366/rimcafd2021.82.013

\title{
ORIGINAL
}

\section{ASOCIACIÓN DE ÁREAS ESPECIIFICAS DEL DESARROLLO CON DISPRAXIAS EN PREESCOLARES}

\section{ASSOCIATION BETWEEN SPECIFIC AREAS OF DEVELOPMENT AND DYSPRAXIA IN PRESCHOOL CHILDREN}

\author{
Pimenta, R.A. ${ }^{\text {; }}$ Poeta, L.S. ${ }^{2}$; Basso, L. ${ }^{3}$; Mariano, M. ${ }^{4}$ y Rosa Neto, F. ${ }^{5}$ \\ 1 Investigador. Programa de Posgrado en Ciencia del Movimiento Humano. Universidad del \\ Estado de Santa Catarina (Brasil) ricardopimentarp@gmail.com \\ 2 Profesora Doctora del Centro de Deportes. Universidad Federal de Santa Catarina (Brasil) \\ lisianepoeta@hotmail.com \\ 3 Profesor Doctor del Departamento de Pedagogía del Movimiento del Cuerpo Humano, \\ Universidad de São Paulo (Brasil) lucianob@usp.br \\ 4 Investigadora. Departamento de Psiquiatría. Universidad Federal de São Paulo (Brasil) \\ mariano.mrl@gmail.com \\ ${ }^{5}$ Profesor Doctor del Centro de Ciencias de la Salud y del Deporte. Universidad del Estado de \\ Santa Catarina (Brasil) franciscorosaneto@terra.com.br
}

Código UNESCO / UNESCO code: 241004 Desarrollo Humano / Human development

Clasificación Consejo de Europa / Council of Europe classification: 17. Otras: Desarrollo Motor / Motor Development

Recibido 4 de junio de 2019 Received June 4, 2019

Aceptado 22 de noviembre de 2019 Accepted November 22, 2019

\section{RESUMEN}

El objetivo del estudio fue evaluar el desarrollo motor y asociar sus áreas específicas con dispraxias. La muestra fue compuesta por 436 preescolares entre 2 a 5 años. El desarrollo motor fue evaluado en las áreas de Coordinación, Propiocepción y Percepción y los resultados presentados en forma de edad motora y cocientes motores. Los resultados muestran que $57(13,7 \%)$ niños presentaron cociente motor general $\leq 89$, siendo clasificados con dispraxia. La Percepción (orientación espacial y temporal) fue el área con más retrasos (edad motora negativa) y con valores de cociente motor general más bajos. Esta área también presentó correlación positiva con el grupo de riesgo para dispraxia. Estos resultados son relevantes para establecer el perfil de desarrollo motor de los preescolares y así, auxiliar profesores y profesionales en intervenciones y en 
la prevención de problemas futuros del aprendizaje asociados a los retrasos observados.

PALABRAS CLAVE: Preescolares, Desarrollo Infantil, Dispraxia, Percepción, Evaluación Motora

\begin{abstract}
The aim of the study was to evaluate motor development and associate its specific areas with dyspraxia in preschool children. The sample consisted of 436 preschoolers (217 girls and 219 boys) between 2 and 5 years old. Motor development was evaluated in the domains of Coordination, Proprioception and Perception using the Motor Development Scale (EDM) with the results being presented as the motor development age and the motor development quotients. The results show that $57(13.7 \%)$ children have presented general motor quotient $\leq 89$, classified as dyspraxia. Children presented lower scores of general motor quotient and highest developmental delays (greater negative motor age) in the perception area (spatial orientation and temporal orientation). Perception showed a positive correlation with the risk group for dyspraxia. These results are relevant for the establishment of the motor development profile of preschool children and thus, help teachers and professionals in interventions and prevention of future learning problems associated with developmental delays.
\end{abstract}

KEY WORDS: Preschool Child, Child Development, Dyspraxia, Perception, Motor Assessment

\title{
INTRODUCCIÓN
}

La primera infancia se caracteriza por cambios significantes en el desarrollo motor. En esta fase, se torna inherente la adquisición y desempeño de las habilidades de locomoción, estabilización y manipulación, resultado de la interacción entre la herencia genética y las influencias del ambiente en que el niño vive (Shonkoff et al., 2012). Establecer y perfeccionar las habilidades motoras puede tener un impacto positivo en las habilidades cognitivas, optimizando el desarrollo global y la forma de interacción entre el niño y el ambiente (Örnkloo \& Von Hofsten, 2007; Pereira, Valladares, Mieres, Velázquez, \& Pichs, 2019). La adquisición de habilidades motoras proporciona a los niños nuevas oportunidades de aprender a partir de experiencias ambientales, tanto en relación a objetos como a otros individuos (Houwen, Van der Veer, Visser, \& Cantell, 2017).

Investigaciones del desarrollo motor en la educación infantil son fundamentales, pues evaluaciones en edades tempranas pueden identificar niños con riesgo, posibilitando redireccionarlos para futuras intervenciones y minimizar perjuicios relacionados a los retrasos (Maggi, Magalhães, Campos, \& Bouzada, 2014). Es importante reconocer que el compromiso de las habilidades motoras está relacionado con retraso en el desarrollo cognitivo y el lenguaje (Iverson, 2010) y con dificultades sociales y emocionales (Green, Baird, \& Sugden, 2006). En 
complemento, estudios relacionaron retrasos en el desarrollo de habilidades motoras con influencia negativa en los aspectos del individuo durante la vida, como alto índice de obesidad y menor participación en actividad física (Bucco \& Zubiaur, 2015) y un efecto posterior negativo en el desempeño académico de los adolescentes (Kantomaa et al., 2013).

La literatura internacional destaca que $5 \%$ a $6 \%$ de los niños en edad escolar presentan diagnóstico clínico de trastorno motor (American Psychiatric Association [APA], 2013). Sin embargo, estudios recientes en el contexto brasileño muestran que esa prevalencia puede ser mayor. Silva (2017) al evaluar a 265 niños de 3 a 5 años encontró 14,3\%. Maggi et al. (2014) encontraron 19\% de niños con retrasos del desarrollo motor y Valentini et al. (2012), a partir de la evaluación de 152 niños de 4 a 6 años provenientes de los tres estados del Sur del Brasil, encontraron una prevalencia de 35\% con indicación de trastorno motor. Esos compromisos motores pueden ser categorizados como un disturbio por sí solo, variando de un perjuicio motor severo, como en el caso de parálisis cerebral, a menos severo como en niños con Trastorno del Desarrollo de la Coordinación (TDC; Piek, Hands, \& Licari, 2012).

Entre estas disfunciones motoras, la dispraxia se caracteriza como un disturbio del desarrollo neurológico que afecta la capacidad de planear, organizar, coordinar y automatizar gestos orientados (Boxus, Denis, \& Pitchot, 2018). La dispraxia puede ser definida como una perturbación en la planificación de los movimientos intencionales, conscientes y aprendidos (De Ajuriaguerra, 1977), representado una disfunción motora. Cada gesto que no llega a ser automatizado por el niño, exige mayor atención, compitiendo con la ejecución de otras tareas motoras, dejándolo menos disponible. De esta forma, la dispraxia es un trastorno cognitivo de actividad motora y su representación mental, y puede ocurrir no solo en función de los aspectos neurológicos que regulan los factores psicomotores (tales como el tono muscular, control de la postura, lateralidad, organización espacial y temporal, praxis fina y global) como también debido a factores ambientales, familiares, educacionales y sociales (Fonseca, 2009).

Según Baxter (2012), el origen del término se da a partir de la relación con la praxis, donde la dispraxia es descrita como un trastorno relacionado a la dificultad en el aprendizaje y la ejecución de actividades motoras. Las personas con dispraxia presentan como característica una dificultad en combinar actos motores simples para la ejecución de actos motores más complejos, como en la escritura. Además, las expresiones de las dificultades motoras varían desde movimientos de extremidades, como la coordinación ocular-manual, a movimientos amplios como la marcha, control motor facial de labios y lengua y en el habla (Anderson-Mooney, Schmitt, Head, Lott, \& Heilman, 2016). Estos actos motores pueden clasificarse en diferentes áreas del desarrollo motor, como la coordinación, propiocepción y la percepción, y representadas por dominios específicos, por ejemplo, las habilidades motoras gruesas (habilidades como saltar, correr y andar), las habilidades motoras finas (precisión e integración motora fina y destreza manual), el equilibrio, el esquema corporal y la organización espacial y temporal. Esas categorías se asocian a diferentes áreas del desarrollo neurológico y a la necesidad de más o menos atención a intervenciones y estímulos específicos. Por lo tanto, el objetivo de este estudio 
fue evaluar el desarrollo motor de preescolares y asociar los retrasos identificados en áreas específicas del desarrollo con dispraxias.

\section{MATERIAL Y MÉTODOS}

\section{Muestra}

La muestra fue compuesta por 436 niños (217 niñas y 219 niños) sorteados aleatoriamente por conglomerados, en 70 escuelas. Edad comprendida de los niños: 100 preescolares tenían 2 años; 103 con 3 años; 124 con 4 años; y 109 con 5 años. Para estimar el cálculo del tamaño de la muestra, utilizamos como referencia el tamaño de la población de niños matriculados en Educación Infantil de acuerdo con una transferencia del Departamento de Educación Municipal de Florianópolis: 7062 alumnos. Elegimos evaluar el 5\% de los niños en cada etapa de la educación de la primera infancia, considerando un intervalo de confianza del 95\% en Programa de Estadísticas Epidemiológicas de código abierto para la salud pública (OpenEpi; Dean, Sullivan \& Soe, 2013).

Como criterios de inclusión, fueron considerados: a) tener el Término de Consentimiento Libre y Esclarecido (TCLE) firmado por los padres o responsables; b) presentar edad dentro del rango objetivo para el estudio en el momento de la evaluación; c) estar regularmente matriculado en la educación infantil de la red pública municipal de enseñanza de Florianópolis/SC; d) aceptar participar de la investigación y firmar el TCLE en el momento de la evaluación. Como criterio de exclusión, para este estudio se desconsideraron a los niños con diagnóstico clínico de deficiencia (física, visual, auditiva y/o intelectual) y/o trastornos del desarrollo (ejemplo: trastorno del espectro autista).

\section{Instrumento de investigación}

Para evaluar el desarrollo motor de los niños y sus áreas específicas, fue utilizada la Escala de Desarrollo Motor (EDM; Rosa Neto, 2018). La EDM divide la evaluación en tres áreas principales y cada área se compone de dos dominios específicos: coordinación (motricidad fina y motricidad global), propiocepción (equilibrio y esquema corporal) y percepción (organización espacial y organización temporal). Cada niño realiza por lo menos doce tareas motoras proyectadas y condicientes con las edades específicas. Las tareas presentan complejidad que aumentan según la edad, nivel 2 hasta el nivel 11, como presentado en la figura 1. Para la aplicación de las tareas motoras, seguimos las instrucciones del Manual de Evaluación Motora (Rosa Neto, 2018). 


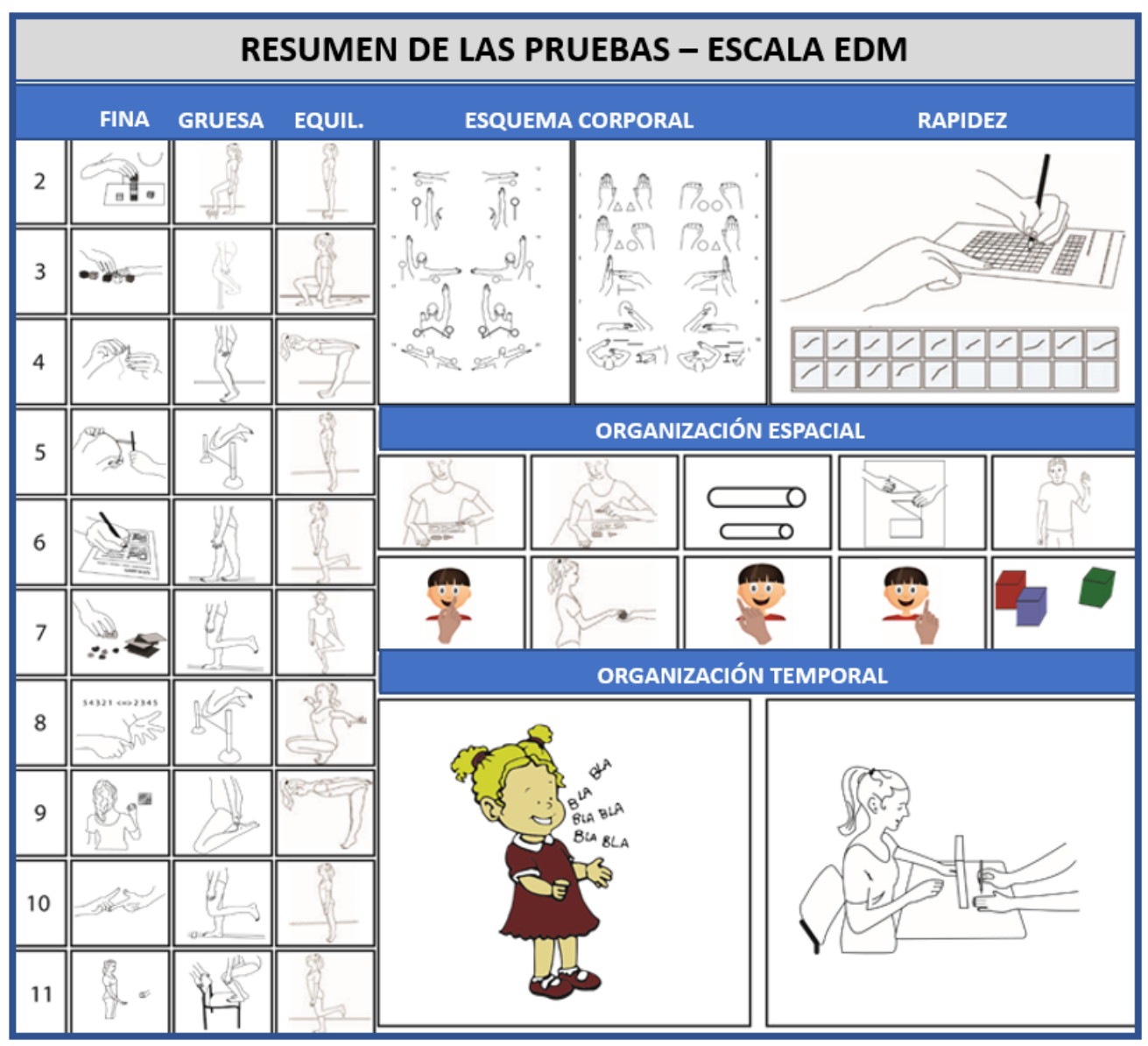

Figura 1. Resumen de las pruebas motoras por edad. Manual de Evaluación Motora (Rosa Neto, 2018).

El instrumento fue estandarizado para evaluar el desarrollo motor en niños a partir de la edad cronológica, edades y cocientes motores, capaz de identificar niños con retrasos que pueden indicar riesgo para el desarrollo motor. La EDM proporciona valores para las edades motoras: promedios de la prueba (resultados expresados em meses) y para cociente motor (edad motora en cada prueba, dividido para la edad cronológica y multiplicado por 100). Los resultados para cocientes motores se refieren a rangos específicos, que clasifican los niveles respectivos de desarrollo, y que varían de "muy bajo" (igual o menos que 69 puntos) a "muy alto" (igual o hasta 130 puntos), como observado en la tabla 1. Los valores menores o iguales a 89 representan riesgo para el desarrollo motor y por ende comprendidos como dispraxia para el estudio en cuestión.

Tabla 1. Clasificación del Cociente Motor General (CMG) según la EDM

\begin{tabular}{ccc}
\hline Puntuación del CMG & Clasificación & Indicador de riesgo \\
\hline 130 o mas & Muy Superior & Ninguno \\
$120-129$ & Superior & Ninguno \\
$110-119$ & Normal Alto & Ninguno \\
$90-109$ & Normal Medio & Ninguno \\
$\mathbf{8 0 - 8 9}$ & Normal Bajo & Riesgo leve \\
$\mathbf{7 0 - 7 9}$ & Inferior & Riesgo moderado \\
$\mathbf{6 9}$ o menos & Muy Inferior & Riesgo grave \\
\hline
\end{tabular}

CMG = Cociente motor general.

Fuente: Manual de Evaluación Motora (Rosa Neto, 2018). 
Los estudios apuntan las propiedades psicométricas adecuadas con respecto a la estandarización de EDM para la población brasileña (Amaro, Santos, Brusamarello, Xavier, \& Rosa Neto, 2009; Rosa Neto, Santos, Xavier, \& Amaro, 2010).

\section{Procedimientos}

Inicialmente el proyecto de investigación fue aprobado por la Secretaria de Educación de la Prefectura Municipal de Florianópolis y posteriormente aprobado por el Comité de Ética en Investigaciones involucrando Seres Humanos (CEISH) de la Universidad del Estado de Santa Catarina (UDESC), bajo el número CAAE 56427116.1.0000.0118. Las escuelas y posteriormente las salas fueron sorteadas por conglomerado, usando Microsoft Excel para hacerlo de forma aleatoria. En las salas sorteadas, los investigadores enviaron los términos de consentimiento a los padres, a través de la agenda escolar de los niños. Una vez que los términos fueron firmados, las escuelas dejaron disponible un lugar separado de los demás niños, donde fueron realizadas las evaluaciones. Las pruebas fueron realizadas por investigadores con entrenamiento y calificados, siempre en pareja (un investigador que condujo la evaluación y otro que registró en la hoja de respuestas del instrumento), dentro de la propia escuela y con duración de 40 minutos por niño.

\section{Análisis de los datos}

Primero se realizó un análisis de prevalencia de dispraxia de la muestra total. Después, fue hecho un análisis de asociaciones entre los grupos normal versus grupo de riesgo para dispraxia, por medio del test de Chi-cuadrado, para las variables sexo, edad y retrasos en las áreas de coordinación, propiocepción y percepción. Finalmente, fue hecho un análisis de correlación entre las áreas del desarrollo, coordinación, propiocepción y percepción entre los grupos normal versus grupo de riesgo para dispraxia, por medio de la correlación de Pearson. Los resultados de los análisis fueron considerados estadísticamente significantes para $p$ valor $\leq 0,05$, con intervalo de confianza de $95 \%$. El análisis de la información se realizó mediante el software estadístico SPSS versión 20.0.

\section{RESULTADOS}

436 preescolares evaluados, $57(13,7 \%)$ presentaron Cociente Motor General $\leq$ 89 (clasificados dentro de la zona de riesgo para el desarrollo), considerados en este estudio como grupo con dispraxias. La Tabla 2 presenta datos descriptivos de la evaluación motora realizada y la prevalencia de dispraxia. 
Tabla 2. Valores descriptivos de la muestra en relación a edad motora general, cocientes motores y clasificación del desarrollo motor.

\begin{tabular}{|c|c|c|c|c|c|c|c|c|c|c|c|c|}
\hline & \multicolumn{6}{|c|}{ Desarrollo típico $(n=379)$} & \multicolumn{6}{|c|}{ Dispraxias $(n=57)$} \\
\hline & \multicolumn{3}{|c|}{ Masculino $(n=188)$} & \multicolumn{3}{|c|}{ Femenino $(n=191)$} & \multicolumn{3}{|c|}{ Masculino $(n=31)$} & \multicolumn{3}{|c|}{ Femenino $(n=26)$} \\
\hline & $P$ & $\mathrm{De}$ & C & $P$ & $\mathrm{De}$ & C & $P$ & $\mathrm{De}$ & C & $P$ & $\mathrm{De}$ & C \\
\hline EC & 47,42 & 13,59 & - & 48,48 & 12,79 & - & 46,61 & 13,13 & - & 47,35 & 11,93 & - \\
\hline EMG & 50,17 & 13,51 & - & 51,32 & 12,31 & - & 38,45 & 11,11 & - & 39,00 & 10,94 & - \\
\hline CMG & 106,8 & 9,95 & NM & 107,1 & 11,63 & NM & 82,5 & 6,06 & NB & 81,92 & 7,55 & NB \\
\hline CMF & 96,1 & 15,46 & NM & 95,6 & 17,04 & NM & 79,2 & 11,81 & 1 & 73,82 & 11,34 & I \\
\hline CMGI & 117,9 & 20,08 & NA & 117,2 & 21,24 & NA & 95,5 & 13,45 & NM & 91,87 & 12,79 & NM \\
\hline CMEq & 106,4 & 17,42 & NM & 106,9 & 18,86 & NM & 82,9 & 11,10 & NB & 86,56 & 15,30 & NB \\
\hline CMEC & 109,4 & 17,78 & NM & 110,6 & 17,36 & NA & 82,0 & 14,37 & NB & 82,76 & 14,31 & NB \\
\hline CMOE & 108,5 & 19,97 & NM & 108,9 & 19,92 & NM & 86,9 & 20,98 & NB & 82,48 & 16,98 & NB \\
\hline CMOT & 102,3 & 18,89 & NM & 103,6 & 22,99 & NM & 68,7 & 20,85 & MI & 74,01 & 15,02 & I \\
\hline
\end{tabular}

$\mathrm{P}=$ promedio; $\mathrm{De}=$ Desviación estándar; $\mathrm{C}=$ Clasificación del desarrollo motor; $\mathrm{EC}=$ Edad cronológica en meses; $\mathrm{EMG}=$ Edad motora general en meses; $\mathrm{CMG}=$ Cociente motor general; CMF = Cociente motor motricidad fina; $\mathrm{CMGI}=$ Cociente motor motricidad global; $\mathrm{CMEq}=$ Cociente motor equilibrio; $\mathrm{CMEC} \mathrm{=}$

Cociente motor esquema corporal; $\mathrm{CMOE}=$ Cociente motor organización espacial; $\mathrm{CMOT}=\mathrm{Cociente}$ motor organización temporal; NA = Normal Alto; NM = Normal Medio; NB = Normal Bajo; I = Inferior; $\mathrm{MI}=$ Muy Inferior.

Podemos observar que además de los valores más bajos encontrados, es posible observar edades motoras negativas en relación a edad cronológica en el grupo dispraxia. Para la muestra en general, las áreas con puntajes más bajos fueron la motricidad fina y la orientación temporal.

La Tabla 3 presenta los resultados del test Chi-cuadrado para verificar la existencia de diferencia estadísticamente significante para el grupo de riesgo para dispraxia entre sexo, edad y meses de retrasos motores comparado con la cronología cronológica.

Tabla 3. Comparación del CMG entre los grupos sexo y edad, EN/EP en las áreas del desarrollo motor

\begin{tabular}{|c|c|c|c|c|}
\hline \multirow{4}{*}{ Sexo } & \multirow{4}{*}{$\begin{array}{l}\text { Niñas } \\
\text { Niños }\end{array}$} & \multicolumn{2}{|c|}{ CMG } & \multirow{4}{*}{$\begin{array}{c}p \\
0,273\end{array}$} \\
\hline & & Normal & Riesgo & \\
\hline & & $191(88 \%)$ & $26(12 \%)$ & \\
\hline & & $188(85,5 \%)$ & $31(14,2 \%)$ & \\
\hline \multirow[t]{4}{*}{ Edad en años } & 2 & $85(85 \%)$ & $15(15 \%)$ & \multirow{4}{*}{0,298} \\
\hline & 3 & $90(87,4 \%)$ & $13(12,6 \%)$ & \\
\hline & 4 & $108(87,1 \%)$ & $16(12,9 \%)$ & \\
\hline & 5 & $96(88,1 \%)$ & $13(11,9 \%)$ & \\
\hline \multirow[t]{4}{*}{ EN/EP Coord } & Edad positiva & $241(86,7 \%)$ & $37(13,3 \%)$ & \multirow{4}{*}{0,300} \\
\hline & $\mathrm{EN}$ - 2 a 6 meses & $103(90,4 \%)$ & $11(9,6 \%)$ & \\
\hline & EN - 7 a 12 meses & $26(78,8 \%)$ & $7(21,2 \%)$ & \\
\hline & $\mathrm{EN}-13$ a 18 meses & $26(78,8 \%)$ & $7(21,2 \%)$ & \\
\hline \multirow[t]{4}{*}{ EN/EP Propio } & Edad positiva & $249(86,8 \%)$ & $38(13,2 \%)$ & \multirow{4}{*}{0,357} \\
\hline & $\mathrm{EN}-2$ a 6 meses & $91(89,2 \%)$ & $11(10,8 \%)$ & \\
\hline & EN - 7 a 12 meses & $33(84,6 \%)$ & $6(15,4 \%)$ & \\
\hline & $\mathrm{EN}$ - 13 a 18 meses & $6(75 \%)$ & $2(25 \%)$ & \\
\hline \multirow[t]{4}{*}{ EN/EP Percep } & Edad positiva & $228(95,4 \%)$ & $11(4,6 \%)$ & \multirow{4}{*}{$\leq 0,00$} \\
\hline & $\mathrm{EN}$ - 2 a 6 meses & $97(77,6 \%)$ & $28(22,4 \%)$ & \\
\hline & EN - 7 a 12 meses & $39(73,6 \%)$ & $14(26,4 \%)$ & \\
\hline & $\mathrm{EN}-13$ a 18 meses & $1(25 \%)$ & $3(75 \%)$ & \\
\hline
\end{tabular}

CMG = Cociente Motor General; EP = Edad motora positiva; EN = Edad motora negativa; Coord = Coordinación; Propio = Propiocepción; Percep $=$ Percepción. 
Puede observar que la diferencia entre edad cronológica y edad motora (EN/EP), apenas retrasos en el área de la percepción presentan diferencias estadísticas entre niños con y sin riesgo de dispraxia.

Se optó por verificar las asociaciones entre los cocientes motores en las áreas de coordinación, propiocepción y percepción, y el grupo de riesgo, por medio de la prueba de correlación de Pearson, como presentado en la tabla 4.

Tabla 4. Correlación entre CMG y EN Coordinación, EN Propiocepción y EN Percepción

\begin{tabular}{ccccc}
\hline & G. Riesgo & EN Coord & EN Propio & EN Percep \\
G. Riesgo & - & 0,029 & 0,022 & $0,246^{* *}$ \\
EN Coord & - & - & $0,582^{* *}$ & $0,423^{* *}$ \\
EN Proprio & - & - & - & $0,484^{* *}$ \\
EN Percep & - & - & - & - \\
\hline \multirow{4}{*}{ Correlación significante $p$ valor $\leq 0,00 ;$ EN = edad Negativa; Coord = Coordinación; Propio $=$} \\
\multicolumn{5}{c}{ Propiocepción; Percep = Percepción; G. Riesgo = Grupo de Riesgo }
\end{tabular}

Edad motora negativa en la Coordinación, Propiocepción y Percepción son las variables que se alteran de forma directamente proporcional, a medida que, mientras más retrasos un niño presenta en alguna de las áreas, mayores serán los déficits en las demás áreas. Los resultados del test de correlación presentados en la Tabla 4 también indican que la edad negativa en el área de la percepción puede impactar directamente el riesgo de dispraxia en preescolares, algo que no fue observado en la Coordinación y Propiocepción.

\section{DISCUSIÓN}

Con el objetivo de evaluar el desarrollo motor de preescolares y asociar retrasos identificados con dispraxias, el presente estudio evaluó la coordinación motora (motricidad fina y global), la propiocepción (equilibrio y esquema corporal) y la percepción (orientación en el tiempo y espacio) de 436 niños de dos a cinco años regularmente matriculados y frecuentando la educación infantil de la red municipal de enseñanza de Florianópolis-SC.

Los resultados de la evaluación motora muestran que $13,7 \%$ de los niños presentan dispraxias (cociente motor general $\leq 89$ ) y los mayores indicios de retrasos motores (niños clasificados como "normal bajo", "inferior" o "muy inferior") fueron observados en el área de la percepción, en los dominios de la orientación espacial y temporal. También fue observado que las tareas que exigen una mayor carga de los componentes cognitivos, como la motricidad fina, la orientación espacial y la organización temporal, presentaron promedio y valores mínimos más bajos que los encontrados para las tareas de motricidad global y equilibrio.

Mientras menor es la edad del niño, más relevante es el retraso observado, comprendiendo que los primeros meses y años de vida son fundamentales para su desarrollo. En ese primer periodo de la vida es cuando se observa más plasticidad neural, o sea, mayor capacidad del sistema nervioso central para organizar, reorganizar y adaptar las redes neuronales en respuesta a los 
requisitos de las tareas y a las demandas del ambiente, así como en el desarrollo de demandas denominadas internas (Johnston, 2009). Estos desordenes o retrasos motores pueden interferir en la madurez global del niño, afectando el desarrollo de sus relaciones sociales, ejecución de las tareas de vida diaria, aspectos cognitivos, aprendizaje y lenguaje (Lamônica \& Picolini, 2009). Araújo (2013) al acompañar el desarrollo psicomotor de 0 a 3 años de 77 niños, encontró $31,2 \%$ de los sujetos en la zona de riesgo para el desarrollo, donde el área del lenguaje tenía el mayor índice de retrasos (57,5\%). Caetano, Silveira y Gobbi (2005) observaron valores inferiores para la motricidad fina y organización espacial en la evaluación motora de 30 niños de 3,4 y 5 años.

Sobre el área de la percepción (organización espacial y temporal) en particular, fueron observados los mayores retrasos (con niños presentando retrasos - edad negativa - de hasta 24 meses), así como observado que esa área puede impactar directamente el riesgo de dispraxia en preescolares. Como definición, el desarrollo de la organización espacial y temporal implica en la capacidad de representar mentalmente las relaciones espaciales y anticipar el curso y el resultado de las transformaciones aplicadas a esas relaciones, es esperado que los niños con desarrollo típico sean capaces de usar informaciones de distancia y de puntos de referencia para definir lugares, posiciones y tamaños (Rosser, 1994). La organización temporal, representada en este estudio por el lenguaje, tiene una estrecha relación con los disturbios motores y el aprendizaje. Hill (2001) en su revisión de literatura sobre estudios que investigaron niños con comprometimiento especifico del lenguaje, encontró que de 40 a $90 \%$ de estos también presentaban deficiencias motoras, complementando, Webster, Majnemer, Platt y Shevell (2005) encontraron en niños diagnosticados con disturbios de lenguaje a los 3 años, asociaciones posteriores (después de los 4 años) con las dificultades motoras y cognitivas.

El compromiso de la orientación espacial y temporal puede estar relacionado a una disfunción de la corteza prefrontal, lo que causa déficits en la organización, memoria de trabajo y atención; y a una disfunción del cerebelo - tálamo - lo que puede causar déficits para inhibición y control motor (Goulardins, Marques, Casella, Nascimento, \& Oliveira, 2013; Rosa Neto, Goulardins, Rigoli, Piek, \& Oliveira, 2015). Del punto de vista neurológico, la corteza prefrontal, los ganglios de la base y el cerebelo son activados para tareas motoras y cognitivas (Diamond, 2000). La corteza prefrontal ha sido asociada desde hace mucho tiempo a la capacidad cognitiva y el cerebelo con la capacidad motora, sin embargo, estudios de casos de pacientes con daños cerebrales localizados, junto con estudios de neuroimagen de individuos con funcionamiento típico, ofrecen evidencias de una superposición entre las dos estructuras cerebrales y los dos tipos de habilidad (Carlson, Rowe, \& Curby, 2013; Diamond, 2000).

Estos hallazgos sobre dispraxias observadas en áreas motoras específicas nos remiten a la importancia de ampliar la atención a las diversas áreas y dominios motores en la educación infantil, ya que la literatura demuestra que los retrasos del desarrollo motor pueden indicar perjuicios en el desempeño académico y hasta trastornos de aprendizaje en la educación primaria (Westendorp, Hartman, Houwen, Smith, \& Visscher, 2011). Cabe destacar que la muestra fue formada por niños de hasta 5 años y 11 meses, lo que es compatible con el fin de la 
educación infantil e inicio de la educación primaria. Para el ingreso a este nuevo periodo, es necesario que el niño haya desarrollado sus habilidades elementales que servirán de base para el aprendizaje de asuntos más complejos. El estudio realizado por Pieters, Desoete, Van Waelvelde, Vanderswalmen y Roeyers (2012) mostró que los niños con dificultades motoras presentan también fracaso escolar en matemáticas, con las habilidades subyacentes a la realización matemática, como memorización y utilización numérica en procedimiento de los cálculos, fueron encontrados con retrasos en comparación al grupo control.

Es importante percibir que, especialmente en el periodo preescolar y de entrada a la educación primaria, la coordinación motora y las funciones ejecutivas no existen aisladamente. Se torna esencial considerar la estrecha relación con los procesos espaciales y temporales, así como las relaciones entre sí y con otras habilidades para formar la base de los comportamientos exitosos de los niños en el contexto del aprendizaje especifico, como la resolución de problemas matemáticos, de lectura y escritura (Cameron, Cottone, Murrah \& Grissmer, 2016). Igualmente, hay que considerar que los valores más bajos del desarrollo en los dominios de la organización espacial y temporal representan, además de un índice de retraso motor, perjuicios en la función de atención y memoria, que pueden estar relacionado con disturbios de atención, como el Trastorno de Déficit de Atención e Hiperactividad (TDAH; Gregorio, Pérez, \& Moro, 2019). Poeta y Rosa Neto (2007) encontraron que los niños diagnosticados con TDAH presentan niveles bajos de clasificación para organización espacial y temporal, debido a que las tareas envuelven procesos de localización, orientación, reconocimiento visual-espacial, percepción de distancia y velocidad.

Los niños entre el fin de la educación infantil e inicio a la educación primaria necesitan mostrar que poseen las habilidades fundamentales para la entrada a este nuevo periodo, las cuales son, autorregulación de la sala de clase y habilidades académicas emergentes de alfabetización y numeración. Adquirir competencia con letras y números envuelve reconocer y por fin, reproducir representaciones visuales de conceptos de orden superior (Case \& Okamoto, 1996). Es importante que los niños tengan bien estructuradas las funciones visomotoras para que puedan dedicar más de sus recursos cognitivos a la integración de informaciones conceptuales y perceptivas. Incluso niños de 4 años de edad con control inhibitorio inicial mejoraron adecuadamente en las habilidades de reconocimiento y copia de formas impresas adaptando sus habilidades motoras finas y así contribuyendo y dedicando más atención a las habilidades en las áreas perceptivas ejercidas en el periodo académico (Cameron et al., 2016).

Estar listo para entrar a la educación primaria ha sido tradicionalmente definido por acumular características cognitivas y comportamentales que predicen las realizaciones académicas posteriores y el retraso en las habilidades motoras más complejas pueden reflejar el impacto en el desempeño académico posterior especialmente en la lectura, escritura y en las matemáticas (Pagani \& Messier, 2012). Las habilidades exigidas en el aprendizaje de la educación primaria, como desempeño en matemáticas y lectura requieren adecuación en dominios complejos perceptivo visuales que deben ser considerados factores más significativos relacionados al desempeño académico. Como observado en este 
estudio, podemos tener desde la educación infantil indicios de retrasos significativos en los dominios de la organización espacial y temporal, considerados predictores del desempeño académico en edades posteriores.

Tal como relatado referente a las habilidades motores finas, los estudios han apuntado la estrecha relación entre las funciones ejecutivas y la organización en el espacio. Verdine, Irwin, Golinkoff y Hirsh-Pasek (2014) apuntaron que las habilidades espaciales asociadas a la función ejecutiva representaron cerca de $70 \%$ de la variación en el desempeño matemático inicial y final de los niños. Estos datos son consistentes con la hipótesis de que la habilidad espacial puede mejorar el desarrollo infantil del conocimiento numérico, ayudándolos a adquirir una representación espacial lineal de números (Gunderson, Ramirez, Beilock, \& Levine, 2012).

Aparte del área de percepción, comúnmente estudios señalan la relación entre una menor habilidad motora en las áreas de la coordinación con los trastornos de aprendizaje (Gomez et al., 2015; Jongmans, Smits-Engelsman, \& Schoemaker, 2003) y otros trastornos específicos, como la dislexia (Biotteau, Chaix, \& Albaret, 2015; Biotteau et al., 2017). El desarrollo de la coordinación motora fue relacionado a los retrasos en la lectura y en las habilidades matemáticas, como reportado por Westendorp et al. (2011) donde los niños con dificultades de aprendizaje obtuvieron resultados más bajos en las pruebas de locomoción y control amplio de objetos en relación al grupo control con desarrollo típico, presentando una relación específica entre lectura y habilidades locomotoras y una tendencia para una relación entre matemáticas y habilidades de control amplio de objetos. En niños brasileros, Padilha, Seidel y Copetti (2014), encontraron que $15,91 \%$ de la muestra de preescolares estaba abajo del promedio en el área de motricidad global.

Sin embargo, a pesar de esta relación con los disturbios de aprendizaje, los dominios más globales, parecen estar más relacionados con las competencias sociales y el bienestar físico de los niños, funcionando como una puerta de entrada para el desarrollo en las actividades físicas y sociales, incluyendo deportes y juegos, durante los años escolares (Caçola, 2014; Pagani \& Messier, 2012). Los niños con retrasos significativos de la coordinación motora, designados como niños con Trastornos en el Desarrollo de la Coordinación (TDC), están más propensos a no participar de actividades físicas comparados al grupo control, explicando la ligación entre las habilidades motoras y las sociales (Wilson, Piek, \& Kane, 2013). Niños con dificultades motoras pueden ser rechazados socialmente o carecen de confianza para participar en deportes o juegos, así como presentan dificultades para participar en actividades típicas de la infancia (como andar en bicicleta, por ejemplo) y de esta forma se tornan más sedentarios y aislados socialmente (Caçola, 2014).

Finalmente se resalta que la evaluación e intervención motora a temprana edad tiene como objetivo principal aumentar los niveles de habilidad en todos los dominios del desarrollo motor para prevenir o minimizar retrasos y consecuencias secundarias, como problemas emocionales y académicos. Destacando la importancia de los profesionales involucrados en la atención a los niños de la educación infantil, Mazeau (2010) afirma que en condiciones de 
identificación precoz de los desórdenes motores (entre los 4 a 8 años de edad) e intervención bien ejecutada por la propia escuela, el pronóstico de los niños es excelente en términos de escolaridad, elección de profesión en el futuro e inserción social, sin embargo, en caso de que esto no ocurra, o si la dispraxia no es aislada (por ejemplo, está asociada a dislexia, hiperactividad, déficit de atención y/o características psicóticas), el niño debe ser encaminado y acompañado también por profesionales de salud y frecuentar salas de atención educacional especializada.

Como factores limitantes de este estudio, las evaluaciones fueron realizadas en una única oportunidad, imposibilitando evaluar el desarrollo de las habilidades con el pasar del tiempo. Este trabajo científico no investigo los antecedentes de riesgo neurológico (pre, peri y postnatal) y también aquellos niños con dificultades o retraso en el desarrollo. Por fin, la investigación sugiere ampliar la muestra para niños de seis años, de tal forma que sea más condiciente con la edad de ingreso a la educación primaria.

\section{CONCLUSIONES}

Con el objetivo de identificar las dispraxias en la Educación Infantil, valorando áreas específicas del desarrollo motor, el trabajo científico concluye que los dominios de la organización espacial y temporal son los más perjudicados, es decir, los que presentan mayor déficit motor.

También fue observado que las tareas que exigen más de los componentes cognitivos, como la motricidad fina y la orientación espacial y temporal, presentan promedio y valores mínimos más bajos que los encontrados para las tareas de motricidad global y equilibrio.

Además, el estudio destacó que la percepción fue el área en la que los niños presentan más retrasos (edad motora negativa) y menores cocientes motores, y está relacionada con mayor probabilidad de que el niño presente dispraxia. Por tratarse de niños en edad preescolar, es importante reforzar que la adquisición de habilidades motoras está ligada al desarrollo de la percepción del cuerpo, espacio y tiempo, y esas habilidades constituyen componentes de dominio básico tanto para el aprendizaje motor como para las actividades de formación escolar, y que eso refuerza la relación entre el desarrollo motor y cognitivo del niño que al conquistar un buen control motor, también estará construyendo las nociones básicas para su desarrollo intelectual .

Estos resultados son de gran relevancia para establecer un perfil de desarrollo motor de niños de educación infantil y, de esa forma, auxiliar profesores y profesionales del área educacional a entender mejor las áreas y dominios motores con indicadores de retrasos para que estos puedan encontrar puntos clave de desarrollo e intervención de futuros problemas de aprendizaje. El estudio también es una alerta para las relaciones entre los factores de riesgo observados en la educación infantil y el aprendizaje en edades más avanzadas, especialmente en el ingreso a la educación primaria debido al aumento de la exigencia de las habilidades matemáticas, de lectura y escritura. 


\section{REFERENCIAS BIBLIOGRÁFICAS}

Amaro, K. N., Santos, A. P. M., Brusamarello, S., Xavier, R. F. C., \& Rosa Neto, F. (2009). Validação das baterias de testes de motricidade global e equilíbrio da EDM. Revista Brasileira de Ciência e Movimento, 17(2).

American Psychiatric Association. (2013). Diagnostic and Statistical Manual of Mental Disorders. (5th ed.) Washington: American Psychiatric Association. https://doi.org/10.1176/appi.books.9780890425596

Anderson-Mooney, A. J., Schmitt, F. A., Head, E., Lott, I. T., \& Heilman, K. M. (2016). Gait dyspraxia as a clinical marker of cognitive decline in Down syndrome: A review of theory and proposed mechanisms. Brain and Cognition, 104, 48-57. https://doi.org/10.1016/j.bandc.2016.02.007

Araujo, L.B. (2013). Análise do desenvolvimento neuropsicomotor de crianças de zero a três anos em centros de educação infantil. (Dissertação de Mestrado). Universidade Federal do Paraná.

Baxter, P. (2012) Developmental coordination disorder and motor dyspraxia. Developmental Medicine and Child Neurology, 54(1), 3-3. https://doi.org/10.1111/j.1469-8749.2011.04196.x

Biotteau, M., Chaix, Y., \& Albaret, J.-M. (2015). Procedural learning and automatization process in children with developmental coordination disorder and/or developmental dyslexia. Human Movement Science, 43, 78-89. https://doi.org/10.1016/j.humov.2015.07.005

Biotteau, M., Péran, P., Vayssière, N., Tallet, J., Albaret, J.-M., \& Chaix, Y. (2017). Neural changes associated to procedural learning and automatization process in Developmental Coordination Disorder and/or Developmental Dyslexia. European Journal of Paediatric Neurology, 21(2), 286-299. https://doi.org/10.1016/j.ejpn.2016.07.025

Boxus, N., Denis, C., \& Pitchot, W. (2018). Vignette diagnostique de l'étudiant Le point sur la dyspraxie développementale chez l'enfant. Rev Med Liege, 73(3), 156-161.

Bucco, L. y Zubiaur, M. (2015). Análisis del desarrollo motor en escolares brasileños con medidas corporales de obesidad y sobrepeso. Revista Internacional de Medicina y Ciencias de la Actividad Física y el Deporte, 15(59), 593-611. https://doi.org/10.15366/rimcafd2015.59.012

Cacola, P. (2014). Movement Difficulties Affect Childrens Learning: An Overview of Developmental Coordination Disorder (DCD). Learning Disabilities: A Multidisciplinary Journal, 20(2), 98-106. https://doi.org/10.18666/LDMJ2014-V20-I2-5279

Caetano, M. J. D., Silveira, C. R. A., \& Gobbi, L. T. B. (2005). Desenvolvimento motor de pré-escolares no intervalo de 13 meses. Revista Brasileira de Cineantropometria e Desempenho Humano, 7(2), 05-13.

Cameron, C. E., Cottone, E. A., Murrah, W. M., \& Grissmer, D. W. (2016). How Are Motor Skills Linked to Children's School Performance and Academic Achievement? Child Development Perspectives, 10(2), 93-98. https://doi.org/10.1111/cdep.12168

Case, R., Okamoto, Y., Griffin, S., McKeough, A., Bleiker, C., Henderson, B., \& Stephenson, K. M. (1996). The role of central conceptual structures in the 
development of children's thought. Monographs of the Society for Research in Child Development, 61(1-2). https://doi.org/10.2307/1166077 Carlson, A. G.; Rowe, E.; Curby, T. W. (2013). Disentangling Fine Motor Skills' Relations to Academic Achievement: The Relative Contributions of VisualSpatial Integration and Visual-Motor Coordination. The Journal of Genetic Psychology, 174(5). 514-533. https://doi.org/10.1080/00221325.2012.717122

De Ajuriaguerra, J. (1977). Manual de psiquiatría infantil (4 ed.). Barcelona: Masson S.A.

Dean, A. G., Sullivan, K. M., Soe, M. M. (2013) OpenEpi: Open Source Epidemiologic Statistics for Public Health, www.OpenEpi.com.

Diamond, A. (2000). Close Interrelation of Motor Development and Cognitive Development and of the Cerebellum and Prefrontal Cortex. Child Development, 71(1), 44-56. https://doi.org/10.1111/1467-8624.00117

Fonseca, V. D. (2009). Desenvolvimento psicomotor e aprendizagem. Porto Alegre: Artmed Editora.

Gomez, A., Piazza, M., Jobert, A., Dehaene-Lambertz, G., Dehaene, S., \& Huron, C. (2015). Mathematical difficulties in developmental coordination disorder: Symbolic and nonsymbolic number processing. Research in Developmental Disabilities, 43(44), 167-178. https://doi.org/10.1016/j.ridd.2015.06.011

Goulardins, J. B., Marques, J. C. B., Casella, E. B., Nascimento, R. O., \& Oliveira, J. A. (2013). Motor profile of children with attention deficit hyperactivity disorder, combined type. Research in Developmental Disabilities, 34(1), 40-45. https://doi.org/10.1016/j.ridd.2012.07.014

Green, D., Baird, G., \& Sugden, D. (2006). A pilot study of psychopathology in Developmental Coordination Disorder. Child: Care, Health and Development, 32(6), 741-750. https://doi.org/10.1111/j.13652214.2006.00684.x

Gregorio, M. V., Pérez, L. M. R., \& Moro, M. I. B. (2019). Analysis of the relationships between low motor competence and attention and hyperactivity problems in school age. Retos, 36(36), 625-632. https://doi.org/10.47197/retos.v36i36.68502

Gunderson, E. A., Ramirez, G., Beilock, S. L., \& Levine, S. C. (2012). The relation between spatial skill and early number knowledge: the role of the linear number line. Developmental Psychology, 48(5), 1229-1241. https://doi.org/10.1037/a0027433

Hill, E. L. (2001). Non-specific nature of specific language impairment: A review of the literature with regard to concomitant motor impairments. International Journal of Language and Communication Disorders. 36(2), 149-171. https://doi.org/10.1080/13682820010019874

Houwen, S., van der Veer, G., Visser, J., \& Cantell, M. (2017). The relationship between motor performance and parent-rated executive functioning in 3to 5-year-old children: What is the role of confounding variables? Human Movement Science, 53, 24-36. https://doi.org/10.1016/j.humov.2016.12.009

Iverson, J. M. (2010). Developing language in a developing body: The relationship between motor development and language development. 
Journal of Child Language, 37(2), 229-261. https://doi.org/10.1017/S0305000909990432

Johnston, M. V. (2009). Plasticity in the developing brain: implications for rehabilitation. Developmental disabilities research reviews, 15(2), 94-101. https://doi.org/10.1002/ddrr.64

Jongmans, M. J., Smits-Engelsman, B. C. M., \& Schoemaker, M. M. (2003). Consequences of Comorbidity of Developmental Coordination Disorders and Learning Disabilities for Severity and Pattern of Perceptual-Motor Dysfunction. Journal of Learning Disabilities, 36(6), 528-537. https://doi.org/10.1177/00222194030360060401

Kantomaa, M. T., Stamatakis, E., Kankaanpaa, A., Kaakinen, M., Rodriguez, A., Taanila, A., ... Tammelin, T. (2013). Physical activity and obesity mediate the association between childhood motor function and adolescents' academic achievement. Proceedings of the National Academy of Sciences, 110(5), 1917-1922. https://doi.org/10.1073/pnas.1214574110

Lamônica, D. A. C, \& Picolini, M. M. (2009). Habilidades do desenvolvimento de prematuros. Revista CEFAC, 11(Suppl. 2), 145-153. https://doi.org/10.1590/S1516-18462009005000019

Maggi, E. F., Magalhães, L. C., Campos, A. F., \& Bouzada, M. C. F. (2014). Preterm children have unfavorable motor, cognitive, and functional performance when compared to term children of preschool age. Jornal de Pediatria, 90(4), 377-383. https://doi.org/10.1016/j.jped.2013.10.005

Mazeau, M. (2010). Les dyspraxies: points de repères. Archives de Pédiatrie, 17(3). 314-318. https://doi.org/10.1016/j.arcped.2009.10.016

Örnkloo, H., \& von Hofsten, C. (2007). Fitting objects into holes: On the development of spatial cognition skills. Developmental Psychology, 43(2), 404-416. https://doi.org/10.1037/0012-1649.43.2.404

Padilha, J. F., Seidel, E. J., Copetti, F. (2014). Análise do desenvolvimento motor e qualidade do ambiente domiciliar de crianças pré-escolares. Saúde (Santa Maria), 40(1), 99-108. https://doi.org/10.5902/2236583410763

Pagani, L. S., \& Messier, S. (2012). Links between Motor Skills and Indicators of School Readiness at Kindergarten Entry in Urban Disadvantaged Children. Journal of Educational and Developmental Psychology, 2(1), 95. https://doi.org/10.5539/jedp.v2n1p95

Pereira, L. G., Valladares, L. R., Mieres, A. A. F., Velázquez, L. M. C., \& Pichs, M. A. E. (2019). Influence of early stimulation on the sensorimotor development of children aged four to six: a vision from Karate Do. Retos, (35), 147-155.

Piek, J. P., Hands, B., \& Licari, M. K. (2012). Assessment of Motor Functioning in the Preschool Period. Neuropsychology Review, 22(4), 402-413. https://doi.org/10.1007/s11065-012-9211-4

Pieters, S., Desoete, A., Van Waelvelde, H., Vanderswalmen, R., \& Roeyers, H. (2012). Mathematical problems in children with developmental coordination disorder. Research in developmental disabilities, 33(4), 11281135. https://doi.org/10.1016/j.ridd.2012.02.007

Poeta, L. S., \& Rosa Neto, F. (2007). Motor assessment in school-aged children with indicators of the attention deficit/hyperactivity disorder. Revista de Neurologia, 44(3), 146-149. https://doi.org/10.33588/rn.4403.2005663 
Rosa Neto, F. (2018). Manual de Evaluación Motora - EDM III. Florianópolis, SC: UDESC.

Rosa Neto, F., Goulardins, J. B., Rigoli, D., Piek, J. P., \& Oliveira, J. A. de. (2015). Motor development of children with attention deficit hyperactivity disorder. Revista Brasileira de Psiquiatria, 37(3), 228-234. https://doi.org/10.1590/1516-4446-2014-1533

Rosa Neto, F., Santos, A. P. M., Xavier, R. F. C., \& Amaro, K. N. (2010). A importância da avaliação motora em escolares: análise da confiabilidade da escala de desenvolvimento motor. Revista Brasileira de Cineantropometria \& Desempenho Humano, 12(6), 422-427. https://doi.org/10.5007/1980-0037.2010v12n6p422

Rosser, R. (1994) The developmental course of spatial cognition: Evidence for domain multidimensionality. Child Study Journal, 24(4), 255-280.

Silva, A. P. da (2017). Vulnerabilidade de risco para o desenvolvimento motor: prevalência de dispraxias na Educação Infantil. (Dissertação de Mestrado). Universidade do Estado de Santa Catarina.

Shonkoff, J. P., Garner, A. S., Siegel, B. S., Dobbins, M. I., Earls, M. F., Garner, A. S., ... Wood, D. L. (2012). The Lifelong Effects of Early Childhood Adversity and Toxic Stress. Pediatrics, 129(1), e232-e246. https://doi.org/10.1590/S0103-05822012000300011

Valentini, N. C., Coutinho, M. T. C., Pansera, S. M., Santos, V. A. P. dos, Vieira, J. L. L., Ramalho, M. H., \& Oliveira, M. A. de. (2012). Prevalência de déficits motores e desordem coordenativa desenvolvimental em crianças da região Sul do Brasil. Revista Paulista de Pediatria, 30(3), 377-384. https://dx.doi.org/10.1590/S0103-05822012000300011

Verdine, B. N., Irwin, C. M., Golinkoff, R. M., \& Hirsh-Pasek, K. (2014). Contributions of executive function and spatial skills to preschool mathematics achievement. Journal of Experimental Child Psychology, 126, 37-51. https://doi.org/10.1016/J.JECP.2014.02.012

Webster, R. I., Majnemer, A., Platt, R. W., \& Shevell, M. I. (2005). Motor function at school age in children with a preschool diagnosis of developmental language impairment. The Journal of Pediatrics, 146(1), 80-85. https://doi.org/10.1016/j.jpeds.2004.09.005

Westendorp, M., Hartman, E., Houwen, S., Smith, J., \& Visscher, C. (2011). The relationship between gross motor skills and academic achievement in children with learning disabilities. Research in Developmental Disabilities, 32(6), 2773-2779. https://doi.org/10.1016/j.ridd.2011.05.032

Wilson, A., Piek, J. P., \& Kane, R. (2013). The Mediating Role of Social Skills in the Relationship between Motor Ability and Internalizing Symptoms in Preprimary Children. Infant and Child Development, 22(2), 151-164. https://doi.org/10.1002/icd.1773

Número de referencias totales / Total references: 50 (100\%) Número de referencias propias de la revista / Journal's own references: 1 $(2 \%)$ 